\title{
Workshop on End User Programming for the Web
}

\author{
Allen Cypher \\ IBM Almaden Research Center \\ 650 Harry Rd. MS: B2-422 \\ San Jose, CA 95120 USA \\ acypher@us.ibm.com

\section{Tessa Lau} \\ IBM Almaden Research Center \\ 650 Harry Rd. MS: B2-422 \\ San Jose, CA 95120 USA \\ tessalau@us.ibm.com

\section{Jeff Nichols} \\ IBM Almaden Research Center \\ 650 Harry Rd. MS: B2-422 \\ San Jose, CA 95120 USA \\ jwnichols@us.ibm.com

\section{Mira Dontcheva} \\ Adobe Systems \\ 601 Townsend Street \\ San Francisco, CA 94103 \\ mirad@adobe.com
}

Copyright is held by the author/owner(s)

CHI 2009, April 4 - April 9, 2009, Boston, MA, USA

ACM 978-1-60558-247-4/09/04.

\author{
Keywords \\ End user programming, Web
}

\section{ACM Classification Keywords}

H.5.2] Information interfaces and presentation

\section{Introduction}

In the past several years, there has been a resurgence in research activity in end user programming (EUP), all focused on the web. This work is spread across a variety of institutions and has been published in a variety of conference venues, including CHI, UIST, IUI, and WWW. This workshop will bring these researchers together for a common discussion, with the following goals:

- establish a sense of community amongst researchers in this area;

- discuss common problems and lessons learned about doing research in EUP for the web:

- define a standard corpus of tasks that can be used to evaluate current and future EUP systems; and

- plan the publication of an edited book on the topic of end user programming for the web. 


\section{Why now?}

The timing is right for end user programming to become a standard part of the web browser experience. Today's web users are already experiencing idiosyncratic problems that require adaptive solutions that correspond to each user's particular situation. For example, many users today have web-enabled mobile devices but they still access web pages that were designed to be viewed on desktops. Small screens limit the amount users can see at any given moment, and mobile use often limits how easily users can interact with a device. In this context, removing information irrelevant to the task at hand can be crucial. A second example of idiosyncratic problems-this time on the desktop-arises from web services that enable broader inter-business process chains, which in turn combine web applications in unique ways. This potential explosion of combinations of business applicationseach personalized to an individual office worker's specialized task-cannot possibly be handled by traditional professional programming staff.

End user programming is a way to address this need for idiosyncratic solutions. Several factors exist today that enable solutions to these problems that were not possible ten years ago:

- As new generations of workers enter the workforce, users are becoming more and more comfortable with interacting with technology and learning to use new applications. As the general level of technical sophistication increases, researchers are increasingly able to employ interface techniquessuch as end user programming-that were previously considered too obscure or complex for the average user.
- The spread of wikis, blogs, online video, and social web applications are making it commonplace for individuals to create new web content, and to share this content with others.

- The standardization of the web platform and the availability of mechanisms for extending web browsers make it possible to instrument browsers to record and interpret the user's interaction with web-based applications, providing the data necessary for EUP systems.

\section{Current EUP research}

Researchers in this area have been pursuing a number of different domains, including user-driven web task automation $[8,9,10]$, mash-ups $[1,2,3,5,6,7]$, summarization [4], and mobilization [11]. There are a variety of problems that arise across these domains, and this workshop will enable researchers from different domains to compare insights into these problems.

For instance, several systems (e.g. Chickenfoot [9], Creo [10], and CoScripter [11]) have been designed to enable users to capture and automate repetitive tasks on the web. These systems enable users to script interactions with one or more web sites in order to automatically perform those interactions in the future.

However, the web is a dynamic place, and pages can change over time. How can a script reference the same element on a page despite changes in the structure of that page over time? A very similar problem arises in web summarization systems, such as [4]. Web summarization work has focused on helping people create their own collections and views of web content 
semi-automatically. Suppose a user of one of these systems selects elements of interest on a web page about a hotel. How does the system find the analogous elements on a page for a different hotel? This workshop will enable researchers in task automation and web summarization to compare their approaches to determining page similarity.

A second shared problem is to infer a user's intent. In systems that rely on users' demonstrations to author scripts, (such as Creo[10] and CoScripter[11]), generalization from these demonstrations remains an open challenge. Did a user intend to enter the literal date July $14^{\text {th }}$, tomorrow's date, or the second Wednesday of the month? Inferring the correct generalization from a small number of examples might benefit from the use of commonsense knowledge not present in today's applications.

\section{Workshop format}

The workshop will begin with brief presentations in the morning and discussion sessions in the late morning and afternoon. All participants will be required to read all accepted position papers prior to the start of the workshop. A small subset (four to six) of the accepted papers will be selected for brief 15-minute oral presentations at the workshop. Following the initial presentations, the remainder of the workshop will be devoted to discussion on the following topics: scenario and task corpora, sharing resources, and planning for the edited book.

\section{Scenario and task corpora}

"Visionary" scenarios, such as Apple's Knowledge

Navigator video or Bush's Memex, can serve to inspire generations of research towards a common goal. What are the analogous visionary scenarios for the field of end user programming? If we were to imagine a future in which end user programming were commonplace, what would those systems be capable of, and how would we interact with them? In this discussion, we will collaboratively sketch out a vision for the future of research in this field.

As a field matures, there is a growing need for standardized ways to measure progress. Towards this goal, we will devote part of the workshop to producing a shared corpus of tasks that can be used to evaluate and compare current and future EUP systems.

\section{Sharing resources}

Many of the challenges related to building robust and scalable EUP systems are shared by all researchers who wish to make progress in this area. For example, as noted above, most web-based EUP systems have a need to identify elements on web pages in a way that is robust to changes in the web page structure or the web site design, and that is robust to the differences in analogous web pages.

Solutions to these problems are continually being reinvented by researchers attempting to build EUP systems on the web. In addition to sharing knowledge about common approaches to these problems, this part of the workshop will investigate the possibility of creating a common research platform to which everyone can contribute code and leverage each others' work.

Book planning

The conclusion of the workshop will focus on organizing the authoring of an edited book on "End User 
Programming for the Web". The organizers will be responsible for finding and selecting a publisher (e.g., MIT Press, Springer, and Morgan Kaufmann). Deadlines for first, second, and final drafts of chapters will be set; a method for ensuring substantive reviews and critiques will be established, as well as deadlines for reviews. The schedule will be arranged so that the book will be published before $\mathrm{CHI}$ 2010. Finally, participants will volunteer for chapters that they want to write, and they will agree to adhere to the established deadlines.

\section{About the organizers}

Allen Cypher organized a workshop in 1992 that lead to the creation of the book "Watch What I Do: Programming by Demonstration", which was published by MIT Press in 1993.

Tessa Lau has been doing research on end user programming since 1997, resulting in more than a dozen technical papers on the various aspects of EUP. She also contributed a chapter about her SMARTedit system to the second EUP book, "Your Wish Is My Command".

Jeffrey Nichols is interested in leveraging end user programming techniques to create better experiences for mobile users. He currently leads the Highlight project, which is building technologies to allow end users to create mobile versions of existing web pages.

Mira Dontcheva is interested in creating systems that allow users to access the information they need quickly and easily. Her dissertation work was on interaction techniques for semi-automatic gathering and customization of Web content.

\section{References}

[1]Dapper http://www.dapper.net/

[2]Yahoo Pipes http://pipes.yahoo.com/

[3]Ennals, R., Gay, D., User-friendly functional

programming for the web mashups. In ICFP 2007:

Proceedings of the 2007 ACM SIGPLAN international conference on Functional programming, ACM Press, pages 223-234.

[4]Dontcheva, M., Drucker, S. M., Salesin, D., and Cohen, M. F. Relations, cards, and search templates: user-guided web data integration and layout. In Proceedings of UIST '07. ACM, New York, NY, 61-70.

[5]Hartmann, B., Wu, L., Collins, K., and Klemmer, S. R., Programming by a sample: rapidly creating web applications with d.mix. Proceedings of UIST '07. ACM, New York, NY, 241-250.

[6]Huynh, D., Mazzocchi, S., and Karger, S., Piggy bank: Experience the semantic web inside your browser. In ISWC'05: Proceedings of the 4th International Semantic Web Conference, 2005.

[7]Wong, J. and Hong, J., Marmite: end-user programming for the web. Proceedings of $\mathrm{CHI}^{\prime} 06, \mathrm{ACM}$, New York, NY, 1541-1546.

[8]Little, G., Lau, T.A., Cypher, A., Lin, J., Haber, E.M., \& Kandogan, E., Koala: capture, share, automate, personalize business processes on the web. Proceedings of $\mathrm{CHI}^{\prime} 07, \mathrm{ACM}$, New York, NY.

[9]Bolin, M., Webber, M., Rha, P., Wilson, T., and Miller, R.C., Automation and Customization of Rendered Web Pages. Proceedings of UIST '05. ACM, New York, NY, 163-172.

[10]Faaborg, A. and Lieberman, H., A Goal-Oriented Web Browser, Proceedings of CHI'06, ACM, New York, NY.

[11]Nichols, J., Hua, Z., and Barton, J., Highlight: A System for Creating and Deploying Mobile Web Applications, To appear in Proceedings of UIST'2008, ACM, New York, NY. 